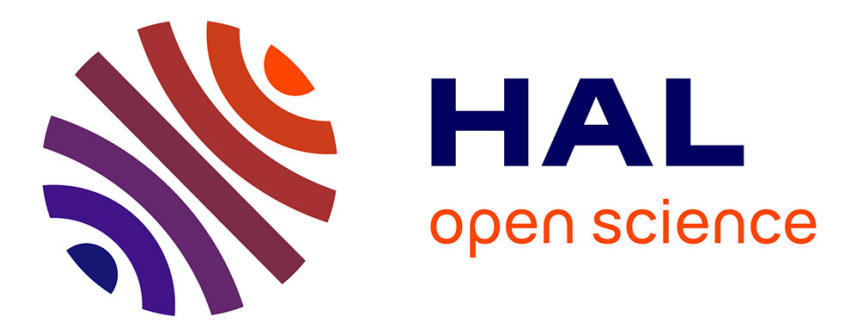

\title{
Spontaneous soft x-ray fluorescence from a superlattice under Kossel diffraction conditions
}

\author{
Philippe Jonnard, Yanyan Yuan, Karine Le Guen, Jean-Michel André, J.-T. \\ Zhu, Z.-S. Wang, Françoise Bridou, Y-y Yuan, J-M André
}

\section{- To cite this version:}

Philippe Jonnard, Yanyan Yuan, Karine Le Guen, Jean-Michel André, J.-T. Zhu, et al.. Spontaneous soft x-ray fluorescence from a superlattice under Kossel diffraction conditions. Journal of Physics B: Atomic and Molecular Physics, 2014, 47 (16), pp.165601. 10.1088/0953-4075/47/16/165601 . hal01058829

\section{HAL Id: hal-01058829 \\ https://hal.science/hal-01058829}

Submitted on 28 Aug 2014

HAL is a multi-disciplinary open access archive for the deposit and dissemination of scientific research documents, whether they are published or not. The documents may come from teaching and research institutions in France or abroad, or from public or private research centers.
L'archive ouverte pluridisciplinaire HAL, est destinée au dépôt et à la diffusion de documents scientifiques de niveau recherche, publiés ou non, émanant des établissements d'enseignement et de recherche français ou étrangers, des laboratoires publics ou privés. 


\title{
Spontaneous soft $x$-ray fluorescence from a superlattice under Kossel diffraction conditions
}

\author{
P. Jonnard*1,2, Y.-Y. Yuan ${ }^{1,2}$, K. Le Guen ${ }^{1,2}$, J.-M. André ${ }^{1,2}$, J.-T. Zhu ${ }^{3}$, Z.-S. Wang ${ }^{3}$, F. \\ Bridou $^{4}$
}

${ }^{1}$ Sorbonne Universités, UPMC Univ Paris 06, Laboratoire de Chimie Physique-Matière et Rayonnement, 11 rue Pierre et Marie Curie, F-75231 Paris cedex 05, France

${ }^{2}$ CNRS UMR 7614, Laboratoire de Chimie Physique-Matière et Rayonnement, 11 rue Pierre et Marie Curie, F-75231 Paris cedex 05, France

${ }^{3}$ Institute of Precision Optical Engineering, Department of Physics, Tongji University, Shanghai 200092, China

${ }^{4}$ Laboratoire Charles Fabry, Institut d'Optique, CNRS UMR 8501, Université Paris Sud 11, 2 avenue Augustin Fresnel, F-91127 Palaiseau Cedex, France

*: corresponding author, e-mail address: philippe.jonnard@upmc.fr

Keywords: superlattice, multilayer, $\mathrm{x}$-ray emission, spontaneous emission, $\mathrm{x}$-ray fluorescence, x-ray standing waves

PACS:

The present study gives the proof of principle of a technique that is an extension of Kossel diffraction both from crystals to superlattices and toward the soft x-ray region, allowing the characterization of the interfaces within a periodic structure. We measure the intensity of the $\mathrm{Co} \mathrm{L} \alpha$ and $\mathrm{Mg} \mathrm{K} \alpha$ characteristic fluorescence emissions from a $\mathrm{Mg} / \mathrm{Co}$ superlattice upon soft $\mathrm{x}$-ray excitation. The observation is made so that the angle between the sample surface and the detection direction is scanned around the first and second Bragg peaks of the fluorescence emissions. Clear modulations of the emitted intensities are observed and well reproduced by simulations based on the reciprocity theorem and assuming a perfect stack. The present work gives evidence that such a superlattice plays the role of an optical cavity for the spontaneous emission generated within the stack. This should also be the case for stimulated emission, 
which when combined with pumping free electron laser, will open the road to innovative $\mathrm{x}$ ray distributed feedback lasers. 


\section{Introduction}

The ionization from a core level of an atom can be followed by a spontaneous recombination accompanied by the emission of a characteristic radiation. In the case of a free atom, the duration of this process is the core hole lifetime and the emission is isotropic. When the atom is embedded in a photonic crystal acting as an optical cavity, such as a crystal or a superlattice, spatial modulation of the emitted intensity can occur in certain directions related to the Bragg diffraction of the radiation. This very first interpretation of $\mathrm{x}$-ray diffraction given by M. Laue was established about twenty years later by Kossel[1,2] using electron excitation, leading to the observation of the so-called Kossel lines.

Similar observation has been made more recently using electron excitation in a superlattice, in fact a periodic multilayer[3,4]. The diffraction of the $\mathrm{x}$-ray characteristic radiation produced inside a periodic structure by hard $\mathrm{x}$-rays, electron or ion excitation is now known as Kossel diffraction[5,6]. It is being used mainly to study crystals and their defects[7].

As for $\mathrm{x}$-ray fluorescence, i.e. $\mathrm{x}$-ray emission upon excitation by $\mathrm{x}$-rays, to our knowledge only one set of experiments has been performed on a periodic multilayer with an X-ray tube as a source and a photographic film[8] or a proportional counter[9] as a detector. This study, performed in the hard $\mathrm{x}$-ray region $(0.154 \mathrm{~nm})$, dealt with periodic $\mathrm{Fe} / \mathrm{C}$ multilayers designed for x-ray optics and was interpreted in terms of Kossel lines[10].

The Kossel diffraction technique can be viewed as the reverse of the x-ray standing wave (XSW) techniques[6,11,12] at grazing exit[13] and in multilayers[14]. However, in grazing exit, these techniques are applied to quantify impurities at the top of the structure or in shallow dopants[15]. Applied to multilayers, it is only used in the grazing incidence mode[13,16], generally to probe thin layers deposited on the top of the multilayer[14,17] or to probe the interfaces of the multilayer itself[18].

In this paper we show that it is possible to apply this technique in the soft x-ray range, i.e. for emitted photon having an energy lower than $5 \mathrm{keV}$ or wavelength longer than $0.25 \mathrm{~nm}$. For this purpose, we present here the study of intensity modulation of the synchrotron-excited soft x-ray fluorescence emissions from atoms present in superlattices, when the sample is rotated around the detected direction corresponding to the Bragg diffraction of the emitted radiation. In this case, the superlattice sample is made of a periodic alternation of bi-layers, each layer having a nanometer thickness. The advantage of using a superlattice is that its period can be designed to fit particular angular and spectral ranges. Moreover, observations in the soft $x$-ray range, where optical constants are sensitive to the chemical composition can indicate whether interdiffusion takes place at the interfaces of the stacks and thereby predict 
the optical behaviour of the studied multilayer structures.

\section{Experimental and simulation details}

We show in Figure 1(a) the scheme of our grazing exit fluorescence (GEF) XSW experiment: the sample is rotated so that the glancing angle of detection, i.e. the angle between the sample surface and the x-ray detector, is varied around the Bragg direction of the emitted radiation. In Figure 1(b) we show how a grazing incidence fluorescence (GIF) XSW experiment is performed: the sample is rotated so that the incidence angle, i.e. the angle between the sample surface and the incident beam, is moved around the Bragg direction of the incident radiation.

The experiments were performed with $s$-polarized incident primary radiation on the BEAR beamline[19] of the Elettra synchrotron facility, where the angle between the incident and detection directions is fixed and equal to about $60^{\circ}$ owing to mechanical constraints. This angle is known within $\pm 1^{\circ}$ because it was never precisely measured. Both GEF- and GIFXSW experiments were performed but we focus here only on GEF-XSW results. In the first case, GEF, the angular resolution is governed by the aperture of the x-ray detector. It involved moving away the detector resulting in a low collected intensity. However, this was partly compensated for by using a large divergent incident beam, thus increasing the incident flux. In the second case, GIF, the resolution is governed by the divergence of the incident beam, which is generally quite small on synchrotron beam lines. The detection of the fluorescence radiation was done by using a silicon drift detector cooled to around $-15^{\circ} \mathrm{C}$. In this condition, the spectral resolution is $133 \mathrm{eV}$ at $6.5 \mathrm{keV}$. The angular aperture of the detector was $0.9^{\circ}$.
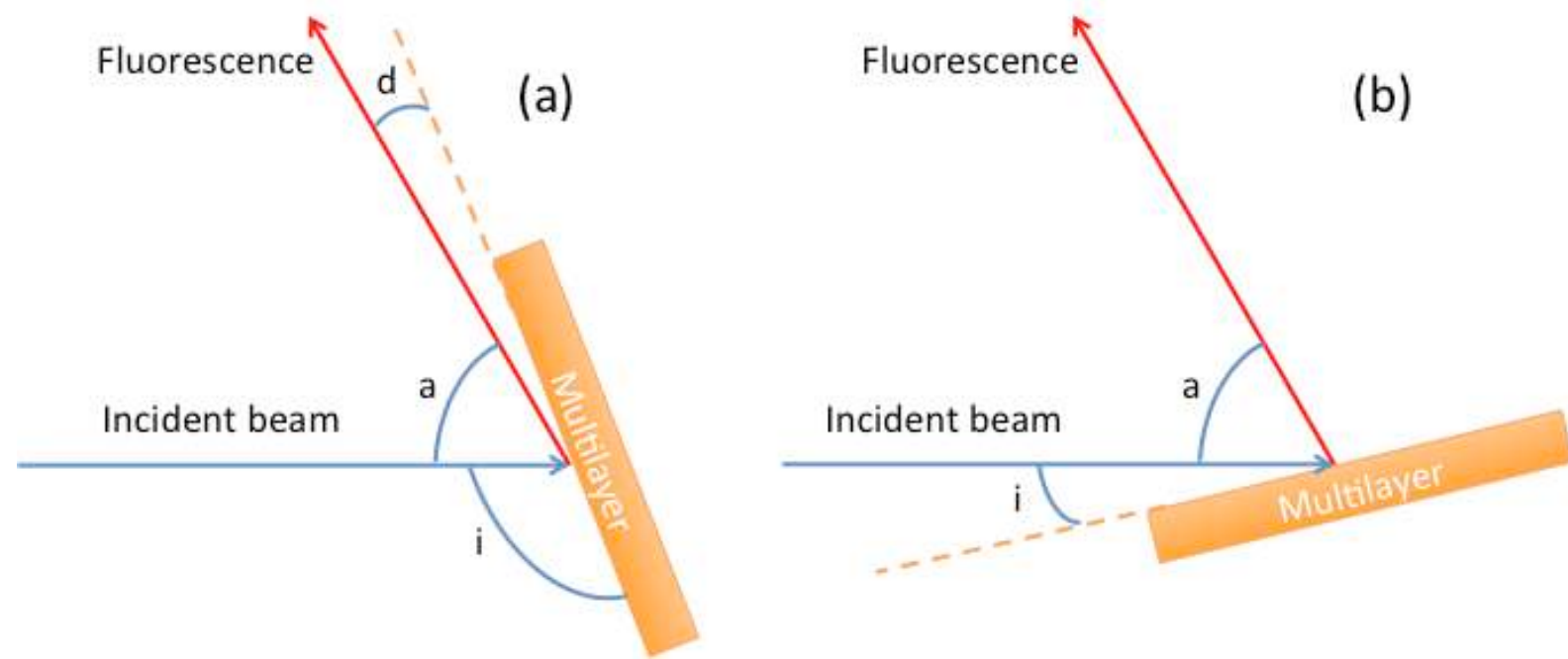

Figure 1: scheme of the x-ray standing wave fluorescence experiments in the grazing exit (a) and incidence (b) modes. Glancing angle of the sample with respect to the incident beam: $i$; angle between the directions of detection and of the incident beam: $a$; detection angle: $d$. 
The simulation model is presented in Ref.[8] and we recall here its main characteristics. In a first step, the intensity of the exciting electromagnetic field, $\left|\mathrm{E}_{\mathrm{ex}}(i, z)\right|^{2}$ at a given depth $z$ in the stack and under a given glancing incidence angle $i$, is calculated. This is done from the recurrent formalism used to calculate the optical properties of stacks from the optical constants and thicknesses of its various layers[14,20]. Then the fluorescence intensity generated at a depth $z$ from an element of $\mathrm{C}(z)$ concentration is proportional to $\mathrm{C}(z)\left|\mathrm{E}_{\text {ex }}(i, z)\right|^{2}$. In a second step, the fluorescence intensity $\left|\mathrm{E}_{\mathrm{fluo}}(d, z)\right|^{2}$ arriving at a detection angle $d$ from a source located at depth $z$ is calculated. This is done by applying the reciprocity theorem, which states that the electric field created in the detector by a dipolar source is the same as the one created at the location of the source by a similar source located at the place of the detector. Since the distance between the source and the detector is very large with respect to the layer thickness, $E_{\text {fluo }}$ is calculated by using the same formalism as the one used to calculate $\mathrm{E}_{\mathrm{ex}}$, assuming a fictive source located at infinity in the detection direction. Finally the intensity of the characteristic fluorescence radiation is proportional to $\mathrm{C}(z)\left|\mathrm{E}_{\mathrm{ex}}(i, z)\right|^{2}\left|\mathrm{E}_{\text {fluo }}(d, z)\right|^{2}$.

The studied superlattice has the following structure and aimed thicknesses: Si substrate / $[\mathrm{Mg}(5.45 \mathrm{~nm}) / \mathrm{Co}(2.55 \mathrm{~nm})]_{\times 30} / \mathrm{B}_{4} \mathrm{C}(3.5 \mathrm{~nm})$. It is denoted "MgCo" in what follows. The thin superficial $\mathrm{B}_{4} \mathrm{C}$ layer prevents the oxidation of the stack. The sample was prepared by magnetron sputtering from high purity, 99.95\%, targets. Its reflectivity curve has been measured in the hard x-ray region $(0.154 \mathrm{~nm})$ and from its fit, the thickness of the different layers and thus the superlattice period was determined. The actual period is $7.78 \mathrm{~nm}$, close to the aimed one, $8.0 \mathrm{~nm}$. Such a system, but with a different period, since designed for x-ray optical applications, has already been characterized[21,22]. From measurements of reflectivity in the hard x-ray and extreme UV regions, of soft x-ray magnetic resonant reflectivity, and of x-ray emission and nuclear magnetic resonance spectroscopies, it was found that the interfaces were abrupt.

\section{Results and discussion}

We show in Figure 2 the $\mathrm{x}$-ray emission spectrum obtained at incident photon energy of $1332 \mathrm{eV}$, that is to say higher than the binding energies of the Co L3 and Mg K levels, i.e. sufficiently high to excite both Co L $\alpha$ ( $3 \mathrm{~d}->2 \mathrm{p}_{3 / 2}$ transition, $\left.776.4 \mathrm{eV}\right)$ and $\mathrm{Mg} \mathrm{K} \alpha\left(2 \mathrm{p}_{3 / 2,1 / 2}-\right.$ $>1 \mathrm{~s}$ transition, $1253,6 \mathrm{eV}$ ) emissions. The spectral resolution of the detector is not enough to 
separate Co L $\alpha$ and Co L $\beta$ emissions. During a measurement, the number of counts under the $\mathrm{Co} \operatorname{L} \alpha$ or $\mathrm{Mg} \mathrm{K} \alpha$ emission is integrated during a given time and plotted as a function of the incident $i$ or detection $d$ angle. From the geometrical scheme of Figure 1(a) it is seen that $d=120-i$ (angles in degrees).

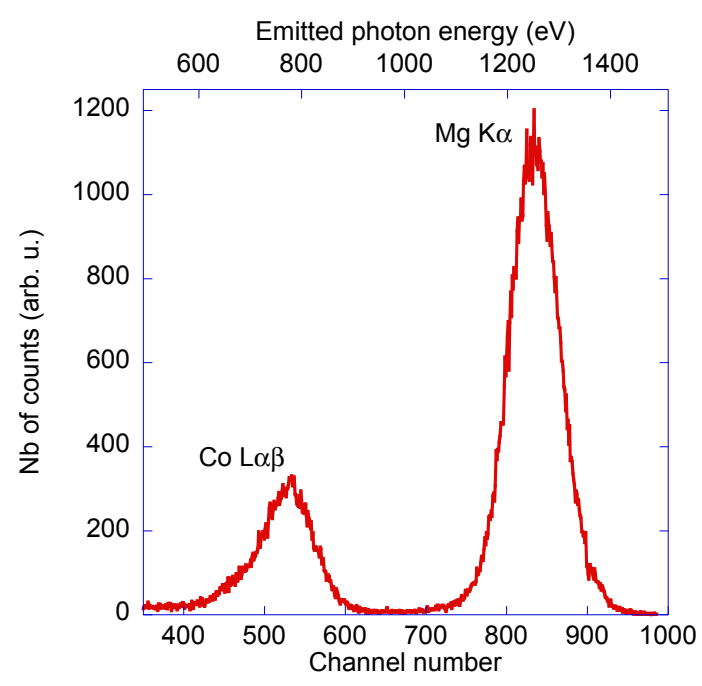

Figure 2: X-ray fluorescence spectrum excited at $1332 \mathrm{eV}$.

For each experiment, at least two different incident photon energies were chosen: 807.6 and $997.6 \mathrm{eV}$ when the Co L $\alpha$ emission was detected; 1332 and $1492 \mathrm{eV}$ when the $\mathrm{Mg} \mathrm{K} \alpha$ emission was detected. The lower values are close to, but above, the absorption threshold; the higher ones are about $200 \mathrm{eV}$ above the threshold. No excitation energy was chosen in close vicinity of the absorption edges in order to avoid the effects of anomalous scattering that generally lead to large variation and uncertainty in the optical index value, making accurate simulations difficult. Moreover, a large divergence of the incident beam makes it less monochromatic, its spectral width varying almost linearly from $6 \mathrm{eV}$ around $810 \mathrm{eV}$ to $12 \mathrm{eV}$ around $1500 \mathrm{eV}$. In the range of the chosen incident photon energies, the optical constants vary slowly and can be considered as constant over the energy width of the incident beam.

We show in Figure 3 the variation of the intensity of the $\mathrm{Co} \operatorname{L} \alpha$ and $\mathrm{Mg} \mathrm{K} \alpha$ emissions as a function of the glancing and detection angles. The number of counts was normalized to the current of a gold mesh inserted in the incident beam in order to correct for its intensity variations. Then, all curves are normalized to the peak maximum in order to compare experiments and simulations. Curves are presented only for the lower incident energies, 807.6 and $1332 \mathrm{eV}$. A modulation of the intensity is clearly observed on each curve at an angle close to the one calculated from the Bragg law in the first diffraction order, for the 
corresponding emitted radiation and corresponding superlattice period. This corresponds to the intercept of the Kossel cone in the detection direction. Owing to the low collected intensity and to the lack of angular resolution, the structures toward large detection angles at an angle corresponding to the second Bragg diffraction order are difficult to observe. The intensities become higher for the lowest photon energies, 807.6 and $1332 \mathrm{eV}$, since the photoionization cross-sections are larger toward the absorption edge. We also note that the shape of the curves is not very sensitive to the incident photon energy. This is why we do not present curves measured at the highest excitation energies.
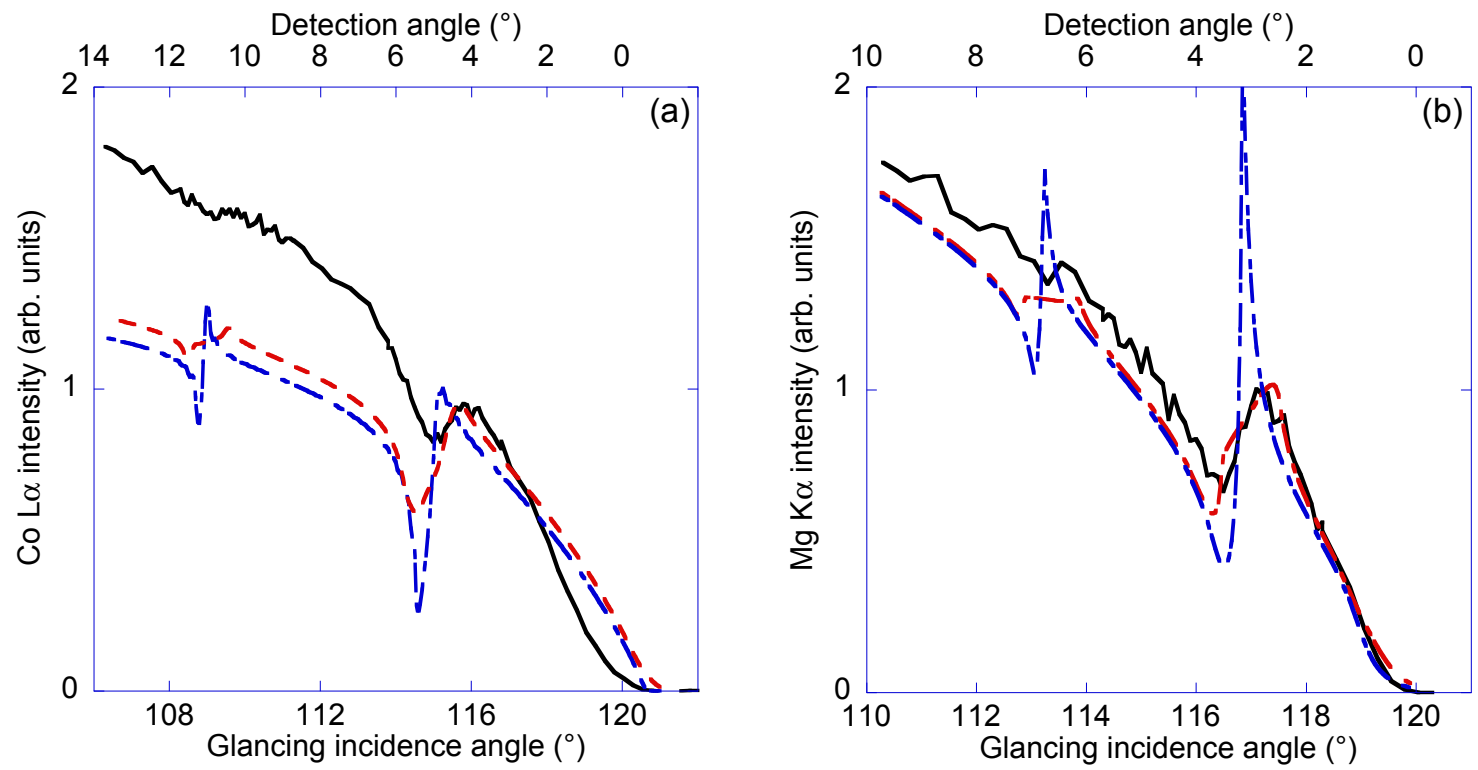

Figure 3: GEF-XSW curves of the MgCo sample, experimental (black solid line) and simulated (dashed lines), obtained at $807 \mathrm{6eV}$ for the Co L $\alpha$ emission (a) and $1332 \mathrm{eV}$ for the $\mathrm{Mg} \mathrm{K} \alpha$ emission (b). The blue dashed line is the un-broadened simulation. The red dashed line is the simulation taking into account the instrumental broadening. Both incident and detection angle scales are indicated.

In Figure 3, we also compare the simulations obtained from the theoretical model[8] with the measurements. The simulations are performed assuming a perfect stack, i.e. without roughness or interdiffusion and without capping layer. No geometrical factor, compensating the variation of the imprint of the incident beam on the sample as a function of the grazing angle[23], has been taken into account since this correction is generally significant only for the very small angles. The initial theoretical data are convoluted with an angular window to account for the instrumental broadening[23]. Moreover a shift of the experimental curves of $0.8^{\circ}$ has been considered in order to put the peak at the same position as in the simulated curves. This can be explained by the uncertainty on the angle between the incidence and detection directions, which should be introduced in the simulation calculations. If the 
broadened simulated curve reproduces relatively well the experimental variation of the $\mathrm{Mg} \mathrm{K} \alpha$ intensity, it gives the general shape of the Co L $\alpha$ intensity variation.

The disagreement between experiment and theory can be attributed mainly to two factors: the difference between the values of the tabulated optical indices and the actual ones and uncertainty of geometrical factors related to polarisation effects. Regarding the first factor, we note that there is a small energy difference, $1.7 \mathrm{eV}$, between the Co L $\alpha$ emission and the Co L3 absorption edge whereas there are about $50 \mathrm{eV}$ between the $\mathrm{Mg} \mathrm{K} \alpha$ emission and the $\mathrm{Mg} \mathrm{K}$ absorption edge. Thus, in the Co case, the optical index is strongly varying in the region of the emission bandwidth, while it is almost constant in the case of $\mathrm{Mg}$. The calculations are made with constant optical indices of the emitted radiation: this does not represent exactly the case of Co emission.

Regarding the second factor, we note that the primary radiation is $s$-polarized and the secondary fluorescence radiation undergoing Bragg diffraction can be decomposed into two linear components of $s$ - and $p$-type in an appropriate reference frame. From the theoretical point of view this decomposition is not a simple task. This complicated operation should be similar to the one employed by Maradudin and Mills for modelling of the scattering by surface roughness[24]. However, we did not perform it in our simulations. In the framework of our model we have calculated the scattering cross-sections of the s-polarisation of the primary radiation towards the $s$ - and $p$-polarisation of the secondary fluorescence radiation. We found that they are practically the same. Since the factors depending on the geometry affecting these two channels (primary $s->$ secondary $s$ and primary $s->$ secondary $p$ ) are different and unknown, there remains in our simulations an element source of uncertainty.

\section{Conclusion}

Diffraction under Bragg condition by superlattices of soft-X-ray fluorescence excited by synchrotron radiation is reported. The Kossel diffraction, applied generally to the study of single crystals, is extended to the case of periodic multilayers. We show that this technique is workable in the soft $\mathrm{x}$-ray range. Moreover, it can complement GIF owing to the large glancing incidence angle, leading to a small footprint of the incident beam on the sample and allowing a localized analysis, depending on the size of the probe beam.

In this proof of principle study, it was possible to approximately reproduce the GEF curves from a periodic $\mathrm{Co} / \mathrm{Mg}$ multilayer by assuming a perfect stack. This confirms the sharpness of the interfaces in this system. However, a better agreement between experiment 
and simulation will be obtained by using correct optical indices and by taking into account the capping layer and polarization effects. In order to avoid or minimize the problem coming from the variation of the optical indices as a function of the incident energy, it would be better, if possible, to work with a characteristic emission whose energy is far from the energy of the involved core level.

This study demonstrates that periodic multilayers designed for the extreme UV and soft x-ray regions can play the role of Bragg reflector for the spontaneous emission generated within the structure. This opens the way for using them as optical cavity for stimulated emission in this spectral domain. Indeed, it is well known from the work by Purcell[25], that the optical cavity affects the transition rate, leading to a suppression or enhancement of the spontaneous emission : the so-called Purcell effect. The control of the spontaneous x-ray emission excited by electrons was discussed in the framework of the Purcell effect in Ref.[26].

This work may have an impact in a number of x-ray techniques and sources, from characterization of the multilayer structure to soft-x-ray radiation sources : x-ray distributed feedback laser (XDFL)[27] or x-ray free electron laser (XFEL) oscillator[28]. Concerning the last point, let us emphasize that soft-X-ray stimulated fluorescence has been recently observed from silicon solid target with pumping by an XFEL[29]. It is reasonable to guess that such a stimulated fluorescence radiation pumped by XFEL could undergo Kossel diffraction in a way similar to the spontaneous one as reported in this paper, opening the way to the realization of an XDFL as proposed by several scientists in the seventies[27,30]. We have calculated[31] that a single XFEL bunch at $57.4 \mathrm{eV}$ delivered by a facility such as FERMI is appropriate to stimulate the $\mathrm{Mg} \mathrm{L} \mathrm{L}_{2,3}$ emission (corresponding to the $3 \mathrm{~s}-3 \mathrm{~d} \rightarrow 2 \mathrm{p}_{1 / 2,3 / 2}$ transition) at $49 \mathrm{eV}$ in a $\mathrm{Mg} / \mathrm{SiC}$ multilayer superlattice forming the distributed laser cavity. The choice of $\mathrm{SiC}$ as spacing layer instead of Co (as in this work) is governed by different reasons, in particular the need to decrease the absorption in the structure. The laser gain threshold is estimated to be around $7910^{3} \mathrm{~cm}^{-1}$. We are convinced that combining superlattices as laser cavity with XFEL for pumping should lead to soft-X-ray DFL in the near future. Implementing the Bragg or Kossel diffraction in the optical scheme of an XFEL oscillator[28] could be envisaged in the soft-x-ray domain although the peak reflectivity of multilayer mirrors in this spectral domain remains relatively low (a few ten percents) compared to the one of "ultimate" crystals for the hard-x-ray range[32,33]. 


\section{Acknowledgments}

This work was done in the framework of the international ANR-NSFC COBMUL project (ANR \#10-INTB-902-01 and NSFC \#11061130549). The research leading to these results has received funding from the European Community's Seventh Framework Programme (FP7/2007-2013) CALIPSO under grant agreement n³12284. Dr. A. Giglia and Pr. S. Nannarone from BEAR beamline of Elettra synchrotron facility are thanked for their help during the synchrotron experiments and their helpful discussions. Dr. N. Spector is thanked for helpful comments.

[1] Kossel W, Loeck V and Voges H 1935 Die Richtungsverteilung der in einem Kristall entstandenen charakteristischen Röntgenstrahlung Z. Physik 94 139-44

[2] Kossel W and Voges H 1935 Röntgeninterferenzen an der Einkristallantikathode Annal. Phys. 415 677-704

[3] Jonnard P, André J-M, Bonnelle C, Bridou F and Pardo B 2002 Modulation of x-ray line intensity emitted by a periodic structure under electron excitation Appl. Phys. Lett. 81 $1524-6$

[4] Jonnard P, André J-M, Bonnelle C, Bridou F and Pardo B 2003 Soft-x-ray Kossel structures from W/C multilayers under various electron ionization conditions Phys. Rev. A 68 032505

[5] Schetelich C, Brenner S and Geist V 1998 Laue and Kossel diffraction on quasicrystals by means of synchrotron radiation J. Synchr. Rad. 5 102-6

[6] Gog T, Bahr D and Materlik G 1995 Kossel diffraction in perfect crystals: X-ray standing waves in reverse Phys. Rev. B 51 6761-4

[7] Langer E and Daebritz S 201075 Years of Kossel patterns - past and future Proc. of the 11th European Workshop of the European-Microbeam-Analysis-Society (EMAS) on Modern Developments and Applications in Microbeam Analysis eds J L Labar, C T Walker, M Zelechower and P Zieba (Bristol: IOP Publishing Ltd) p 012015

[8] Chauvineau J-P and Bridou F 1996 Analyse angulaire de la fluorescence du fer dans une multicouche périodique Fe/C J. Phys. IV 06 C7-53-C7-64

[9] Bridou F, Chauvineau J-P and Mirone A 1998 Étude de la fluorescence du fer dans une multicouche périodique $\mathrm{Fe} / \mathrm{C}$ éclairée sous incidence rasante par un faisceau de rayons $\mathrm{X}$ monochromatique $J$. Phys. IV 08 Pr5-309-Pr5-316

[10] Chauvineau J P, Hainaut O and Bridou F 1996 Lignes de Kossel observées avec des multicouches périodiques Fe/C J. Phys. IV 06 C4-773-C4-779

[11] Gog T, Novikov D, Falta J, Hille A and Materlik G 1994 Kossel diffraction and X-ray standing waves: two birds of one feather J. Phys. IV 04 C9-449-C9-452

[12] Marchesini S, Belakhovsky M, Baron A Q R, Faigel G, Tegze M and Kamp P 1998 Standing waves and Kossel line patterns in structure determination Sol. State Commun. 105 $685-7$

[13] Sakata O and Jach T $2013 \mathrm{X}$-ray standing wave at grazing incidence and exit The $X$ Ray Standing Wave Technique vol 7 (World Scientific) pp 108-21

[14] Bedzyk M J and Libera J A 2013 X-ray standing wave in multilayers The X-Ray Standing Wave Technique vol 7 (World Scientific) pp 122-31

[15] Kayser Y, Banaś D, Cao W, Dousse J -C., Hoszowska J, Jagodziński P, Kavčič M, Kubala-Kukuś A, Nowak S, Pajek M and Szlachetko J 2012 Depth profiling of dopants implanted in Si using the synchrotron radiation based high-resolution grazing emission 
technique $X$-Ray Spectrom. 41 98-104

[16] De Boer D K G 1991 Glancing-incidence x-ray fluorescence of layered materials Phys. Rev. B 44 498-511

[17] Yang S-H, Sell B C, Mun B S and Fadley C S 2013 Probing multilayer nanostructures with photoelectron and $\mathrm{x}$-ray emission spectroscopies excited by $\mathrm{x}$-ray standing waves $T$ The $X$ Ray Standing Wave Technique vol 7 (World Scientific) pp 475-92

[18] Bridou F, Cauchon G and Idir M 2004 Analyse de l'intensité de fluorescence émise par une multicouche périodique sous rayonnement synchrotron. Application à l'étude de la répartition spatiale des éléments dans l'empilement $J$. Phys. IV 118 137-42

[19] Nannarone S, Borgatti F, DeLuisa A, Doyle B P, Gazzadi G C, Giglia A, Finetti P, Mahne N, Pasquali L, Pedio M, Selvaggi G, Naletto G, Pelizzo M G and Tondello G 2004 The BEAR Beamline at Elettra ed T Warwick, J Stohr, H A Padmore and J Arthur AIP Conf. Proc. 705 450-3

[20] Berning P H 1963 Theory and calculations of optical thin films Physics of Thin Films Advances in Research and Development vol 1 (NewYork, London: G. Hass) pp 69-122

[21] Le Guen K, Hu M-H, André J-M, Jonnard P, Zhou S K, Li H C, Zhu J T, Wang Z S and Meny C 2010 Development and Interfacial Characterization of $\mathrm{Co} / \mathrm{Mg}$ Periodic Multilayers for the EUV Range J. Phys. Chem. C 114 6484-90

[22] Le Guen K, Hu M-H, André J-M, Jonnard P, Wang Z, Zhu J, Galtayries A, Meny C, Meltchakov E, Hecquet $\mathrm{C}$ and Delmotte F 2011 Characterization of EUV periodic multilayers $X$-Ray Spectrom. 40 338-42

[23] Li W, Zhu J, Ma X, Li H, Wang H, Sawhney K J S and Wang Z 2012 Geometrical factor correction in grazing incident x-ray fluorescence experiment Rev. Sci. Instrum. 83 053114

[24] Maradudin A A and Mills D L 1975 Scattering and absorption of electromagnetic radiation by a semi-infinite medium in the presence of surface roughness Phys. Rev. B 11 $1392-415$

[25] Purcell E M 1946 Spontaneous emission probabilities at radio frequencies Phys. Rev. $69681-681$

[26] André J-M and Jonnard P 2010 X-ray spontaneous emission control by 1-dimensional photonic bandgap structure Eur. Phys. J. D 57 411-8

[27] Yariv A 1974 Analytical considerations of Bragg coupling coefficients and distributed-feedback x-ray lasers in single crystals Appl. Phys. Lett. 25 105-7

[28] Colella R and Luccio A 1984 Proposal for a free electron laser in the X-ray region Opt. Commun. $\mathbf{5 0} 41-4$

[29] Beye M, Schreck S, Sorgenfrei F, Trabant C, Pontius N, Schüßler-Langeheine C, Wurth W and Föhlisch A 2013 Stimulated X-ray emission for materials science Nature 501 191-4

[30] Fisher R A 1974 Possibility of a distributed-feedback x-ray laser Appl. Phys. Lett. 24 $598-9$

[31] André J-M, Le Guen K and Jonnard P 2014 Feasibility considerations of a soft-x-ray distributed feedback laser pumped by an X-FEL Las. Phys. In press

[32] Durbin S M and Colella R 2010 X-ray optics: Diamond brilliance Nat. Phys. 6 163-4

[33] Shvyd'ko Y V, Stoupin S, Cunsolo A, Said A H and Huang X 2010 High-reflectivity high-resolution X-ray crystal optics with diamonds Nat. Phys. 6 196-9 